\title{
Numerical Estimation of Torsional Dynamic Coefficients of a Hydraulic Turbine
}

\author{
Martin Karlsson, ${ }^{1}$ Håkan Nilsson, ${ }^{2}$ and Jan-Olov Aidanpää ${ }^{3}$ \\ ${ }^{1}$ Lloyd's Register ODS, 10074 Stockholm, Sweden \\ ${ }^{2}$ Department of Fluid Dynamics, Chalmers University of Technology, 41296 Göteborg, Sweden \\ ${ }^{3}$ Division of Solid Mechanics, Department of Mechanical Engineering, Luleå University of Technology,
} 97187 Luleå, Sweden

Correspondence should be addressed to Martin Karlsson, martin.karlsson@lr-ods.com

Received 2 November 2008; Revised 4 March 2009; Accepted 2 April 2009

Recommended by Seung Jin Song

\begin{abstract}
The rotordynamic behavior of a hydraulic turbine is influenced by fluid-rotor interactions at the turbine runner. In this paper computational fluid dynamics (CFDs) are used to numerically predict the torsional dynamic coefficients due to added polar inertia, damping, and stiffness of a Kaplan turbine runner. The simulations are carried out for three operating conditions, one at about $35 \%$ load, one at about $60 \%$ load (near best efficiency), and one at about $70 \%$ load. The runner rotational speed is perturbed with a sinusoidal function with different frequencies in order to estimate the coefficients of added polar inertia and damping. It is shown that the added coefficients are dependent of the load and the oscillation frequency of the runner. This affect the system's eigenfrequencies and damping. The eigenfrequency is reduced with up to $65 \%$ compared to the eigenfrequency of the mechanical system without the fluid interaction. The contribution to the damping ratio varies between $30-80 \%$ depending on the load. Hence, it is important to consider these added coefficients while carrying out dynamic analysis of the mechanical system.
\end{abstract}

Copyright (C) 2009 Martin Karlsson et al. This is an open access article distributed under the Creative Commons Attribution License, which permits unrestricted use, distribution, and reproduction in any medium, provided the original work is properly cited.

\section{Introduction}

Thomas [1] initiated the research on fluid-rotor interactions on turbines in 1958. He suggested an analytical model of destabilising forces due to nonsymmetric clearance in steam turbines. Alford [2] developed a similar model for compressors, where the forces are obtained as a function of the change in efficiency due to increased eccentricity. Urlichs [3] carried out the first research in a test rig and suggested corrections to Thomas and Alford's models. At the same time Iversen et al. [4], Agostinelli et al. [5], and Csanady [6] introduced models of hydraulic unbalance forces due to asymmetry of the flow channel geometry in centrifugal pumps. Hergt and Krieger [7] studied the influence of radial forces during off-design operating conditions. Colding-Jorgensen [8] used potential flow theory to determine damping and stiffness coefficients. Adkins [9] were the first to introduce an analytical model of both mass, damping and stiffness coefficients and harmonic forces. Adkins and Brennen [10], and Bolleter [11, 12] used test rigs to continue the development of models for fluidrotor interactions of pump impellers. Childs [13] used bulk flow theory to determine rotordynamical coefficients at the pump-impeller-shroud surface.

The use of computational fluid dynamics (CFD) has recently increased within the area of fluid-rotor interactions. It was introduced by Dietzen and Nordmann [14] in 1987, but has due to the computational cost not been widely used in the past. The first applications of CFD within rotordynamics have been in the area of hydrodynamic bearings and seals. Recently, CFD has entered into the research of fluid-rotor interactions in centrifugal pumps [15]. CFD has been more common in research and development of hydraulic machinery. Ruprecht et al. $[16,17]$ used CFD to calculate forces and pressure pulsations on axial and Francis turbines. However, the results were not used in rotordynamical analysis. Liang et al. [18] carried out finiteelement fluid-structure interactions of a turbine runner in still water and showed a reduction of the nonrotating 
eigenfrequencies compared to a runner in vacuum. The result had good agreement with the experimental results presented by Rodriguez et al. [19]. Karlsson et al. [20] analyzed the influence of different inlet boundary conditions on the resulting rotordynamic forces and moments for a hydraulic turbine runner. The benefits of using CFD to calculate rotordynamical forces and coefficients of hydraulic turbines have not yet been fully explored. In the present work CFD is used for the determination of the torsional dynamic coefficients due to the flow through the turbine.

\section{Modelling and Simulation}

\subsection{Fluid-Dynamical Model}

2.1.1. The OpenFOAM CFD Tool. In the present work the OpenFOAM (www.openfoam.org) open source CFD tool is used for the simulations of the fluid flow through the Hölleforsen water turbine runner. The simpleFoam OpenFOAM application is used as a base, which is a steadystate solver for incompressible and turbulent flow. It is a finite volume solver using the SIMPLE algorithm for pressurevelocity coupling. It has been validated for the flow in the Hölleforsen turbine by Nilsson [21]. New versions of the simpleFoam application have been developed in the present work, including Coriolis and centrifugal terms and unsteady RANS. All the computations use wall-function grids and turbulence is modelled using the standard $k-\epsilon$ turbulence model. The computations have been run in parallel on 12 CPUs on a Linux cluster, using the automatic decomposition methods in OpenFOAM. The version number used for the present computations is OpenFOAM 1.4.

2.1.2. Operating Conditions. All the computations are made for the Hölleforsen Kaplan turbine model runner, shown in Figure 1. The computational grid is obtained from earlier calculations by Nilsson [21]. The operating conditions used for the present investigations are for runner rotational speeds of $52 \mathrm{rad} / \mathrm{s}, 62 \mathrm{rad} / \mathrm{s}$, and $72 \mathrm{rad} / \mathrm{s}$, which correspond to loads of about $70 \%, 60 \%$, and $35 \%$, respectively. The boundary conditions are kept the same for all operating conditions (in the inertial frame of reference). The change in the load due to the rotational speed is explained by the fact that the pressure drop (or head of the system) needed to drive the same flow through the turbine will change with different rotational speed. The runner rotational speed is finally perturbed with a sinusoidal function in order to identify added coefficients for the torsional dynamic system. This is described below.

2.1.3. Boundary Conditions and Computational Grid. The inlet boundary condition was obtained by taking the circumferential average of a separate guide vane calculation, yielding an axisymmetric inlet flow [22]. This corresponds to a perfect distribution from the spiral casing and without any disturbance from the guide vane wakes.

Wall-functions and rotating wall velocities were used at the walls, and at the outlet the homogeneous Neumann boundary condition was used for all quantities. Recirculating

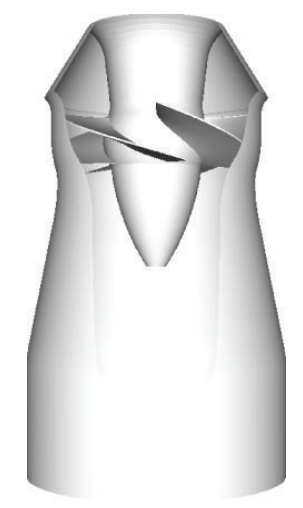

FIgUre 1: The computational domain.

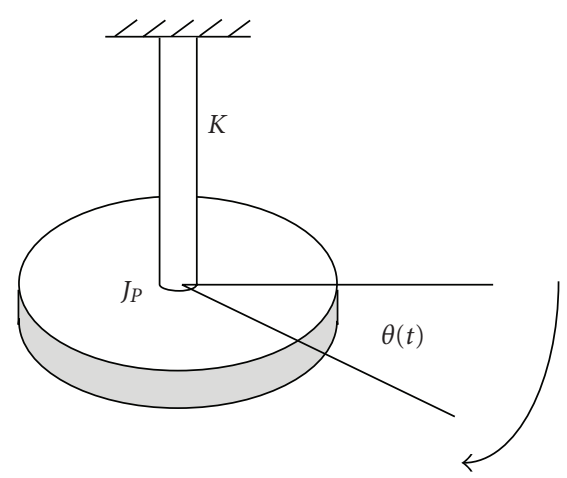

Figure 2: The mechanical model of a torsional dynamic system.

flow was thus allowed at the outlet, and did occur. The turbulence quantities of the recirculating flow at the outlet are unknown, but to set a relevant turbulence level for the present case the back-flow values for $k$ and $\epsilon$ were assumed to be similar to the average of those quantities at the inlet. The background of this assumption is that the turbulence level is high already at the inlet due to the wakes of the stay vanes and the guide vanes. It is thus assumed that the increase in turbulence level is small compared with that at the inlet. It is further believed that the chosen values are of minor importance for the overall flow. For the pressure the homogeneous Neumann boundary condition is used at all boundaries. The computations are made for a complete runner with five blades. The computational domain is shown by Figure 1 . A block-structured hexahedral wall-function grid was used, consisting of approximately 2200000 grid points.

2.1.4. Discretization Schemes. For the convection divergence terms in the turbulence equations the Gamma discretization scheme by Jasak et al. [23] was used. For the convection divergence terms in the velocity equations the GammaV scheme was used, which is an improved version of the Gamma scheme formulated to take into account the direction of the flow field. The Gamma scheme is a smooth and bounded blend between the second-order central differencing $(\mathrm{CD})$ scheme and the first-order upwind differencing (UD) scheme. CD is used wherever it satisfies the 
boundedness requirements, and wherever $\mathrm{CD}$ is unbounded UD is used. For numerical stability reasons, however, a smooth and continous blending between CD and UD is used as $\mathrm{CD}$ approaches unboundedness. The smooth transition between the CD and UD schemes is controlled by a blending coefficient $\beta_{m}$, which is chosen by the user. This coefficient should have a value in the range $0.2 \leq \beta_{m} \leq 1$, the smaller value the sharper switch and the larger value the smoother switch between the schemes. For good resolution, this value should theoretically be kept as low as possible, while higher values are more numerically stable. Studies of different $\beta_{m}$ values have been made, and the results are however more or less unaffected by the choice of $\beta_{m}$. In the present work a value of $\beta_{m}=1.0$ has been used. The time derivative is discretized using the Euler implicit method.

2.2. Identification of Dynamic Coefficients. To describe how the eigenfrequencies and damping of a torsional dynamic system change due to the flow, the model illustrated in Figure 2 is used. In the model, the generator is assumed to be stiff due to the connection to a rigid electric grid, and hence only the torsional motion of the turbine runner is considered. The equation of motion for this system is given by

$$
J_{P} \ddot{\theta}+C \dot{\theta}+K \theta=M(t),
$$

where $J_{P}$ is the polar inertia, $C$ is the damping, $K$ is the stiffness, $M(t)$ an external moment, $t$ is the time, $\theta$ is the angular displacement, $\dot{\theta}$ is the angular velocity, and $\ddot{\theta}$ is the angular acceleration. It is further assumed that the flow through a turbine will give additional inertia, damping, and stiffness to the system. With these additional coefficients the equation of motion becomes

$$
\left(J_{P}+J_{P, \text { Fluid }}\right) \ddot{\theta}+\left(C+C_{\text {Fluid }}\right) \dot{\theta}+\left(K+K_{\text {Fluid }}\right) \theta=M(t),
$$

where $J_{P \text {,Fluid }}$ is the added polar inertia, $C_{\text {Fluid }}$ is the added damping, and $K_{\text {Fluid }}$ is the added stiffness. External moments are negligible $(M(t)=0)$ in the present work. CFD is used to identify the added coefficients from the torque of the turbine runner. Rewriting the moments due to the flow to

$$
T^{\prime}(t)=-J_{P, \text { Fluid }} \ddot{\theta}-C_{\text {Fluid }} \dot{\theta}-K_{\text {Fluid }} \theta,
$$

where $T^{\prime}(t)$ is the total torsional moment due to the flow, and inserting this into (2) yields

$$
J_{P} \ddot{\theta}+C \dot{\theta}+K \theta=T^{\prime}(t) .
$$

To solve $T^{\prime}(t)$, the forces and moments from the CFDsimulations are calculated at each time step. The force on a control volume boundary face is given by

$$
\vec{F}_{\text {face }, i}=p_{\text {face }, i} A_{\text {face }, i} \vec{n}_{\text {face }, i},
$$

where $p_{\text {face, } i}$ is the pressure of the face, $A_{\text {face }, i}$ is the area of the face, and $\vec{n}_{\text {face, } i}$ is the normal vector of the face. The moment of the centre of gravity of the runner at a face is

$$
\vec{M}_{\text {face }, i}=\vec{F}_{\text {face }, i} r_{\text {face }, i},
$$

where $r_{\text {face }}$ is the radius from the centre of gravity to the face. The total moment is calculated as

$$
\vec{M}=\sum_{i=1}^{n} \vec{M}_{\text {face }, i}
$$

where $n$ is the number of faces. The torque is obtained as a scalar product of the moment and the direction vector of the shaft

$$
T(t)=\vec{M} \vec{n}_{y}
$$

During steady conditions the torque is constant in order to provide a constant power to the generator. In case of unsteady conditions, the torque can be written as

$$
T(t)=T_{\text {mean }}+T^{\prime}(t),
$$

where $T_{\text {mean }}$ is the constant part of the torque. In the present work the rotational speed of the turbine runner is prescribed in order to determine the dynamical coefficients of the turbine runner due to the flow. The angular displacement of the runner is given by

$$
\theta=\Omega t+\operatorname{acos}(\vartheta t)=\Omega t+\theta^{\prime},
$$

where $\Omega$ is the constant angular velocity, $t$ is the time, $a$ is an amplitude, $\vartheta$ is a frequency of the prescribed runner oscillation, and $\theta^{\prime}$ is the oscillating part of $\theta$. Below, we are only interested in the oscillating part, where

$$
\theta^{\prime}=\operatorname{acos}(\vartheta t)
$$

gives the velocity

$$
\dot{\theta}^{\prime}=-a \vartheta \sin (\vartheta t)
$$

and the acceleration

$$
\ddot{\theta}^{\prime}=-a \vartheta^{2} \sin (\vartheta t) .
$$

Inserting (11), (12), and (13) into (3) results in an equation for the fluctuation of the torque

$T^{\prime}(t)=a \vartheta^{2} J_{P, \text { Fluid }} \cos (\vartheta t)+a \vartheta C_{\text {Fluid }} \sin (\vartheta t)-a K_{\text {Fluid }} \cos (\vartheta t)$.

This can be written as

$$
T^{\prime}(t)=T_{\mathrm{Amp}} \cos (\vartheta t-\phi)=T_{1} \cos (\vartheta t)+T_{2} \sin (\vartheta t),
$$

where $T_{\mathrm{Amp}}$ is the amplitude of the torque, $\phi$ is the phase angle, and $T_{1}$ and $T_{2}$ are the cosine and sine components of the amplitude. Then the additional damping due to the fluid can be identified as

$$
C_{\text {Fluid }}=\frac{T_{2}}{a \vartheta},
$$

and the additional stiffness and polar inertia due to the fluid can be identified by solving

$$
a \vartheta^{2} J_{P, \text { Fluid }}-a K_{\text {Fluid }}=T_{1}
$$

for two simulations with different values of $\vartheta$. 


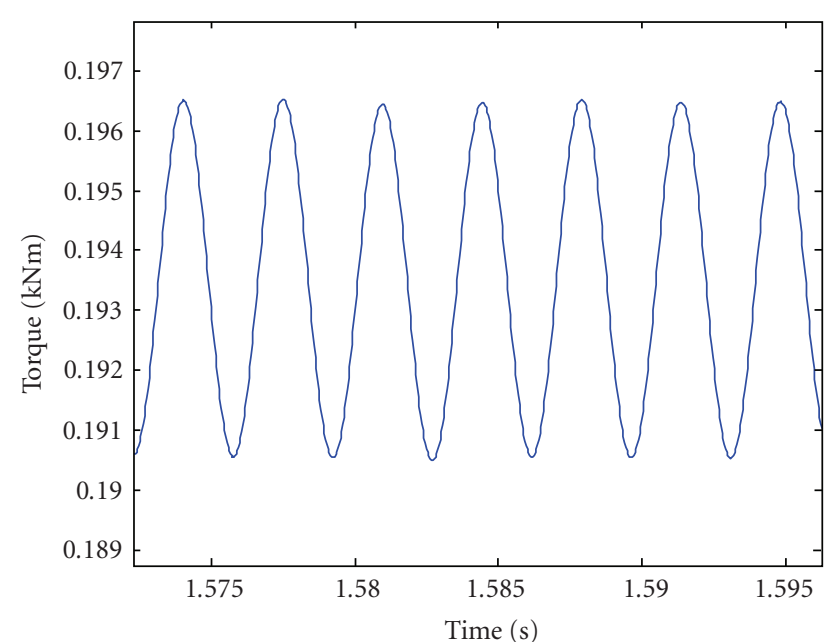

FIgure 3: The torque as a function of time for one of the simulated cases (rotational speed is $72 \mathrm{rad} / \mathrm{s}$ and the oscillating frequency is $1809 \mathrm{rad} / \mathrm{s})$.

The eigenfrequency of (2) can now be solved as

$$
\omega_{D}=\sqrt{\frac{K+K_{\text {Fluid }}}{J_{P}+J_{P, \text { Fluid }}}-\frac{\left(C+C_{\text {Fluid }}\right)^{2}}{4\left(J_{P}+J_{P, \text { Fluid }}\right)^{2}}},
$$

and the corresponding damping ratio is

$$
\zeta=\frac{C+C_{\text {Fluid }}}{2\left(J_{P}+J_{P, \text { Fluid }}\right) \sqrt{\left(K+K_{\text {Fluid }}\right) /\left(J_{P}+J_{P, \text { Fluid }}\right)}} .
$$

\section{Results}

In Figure 3 the torque is shown as a function of time for one of the simulated cases. The amplitude of $T_{1} / a$ in (17) is presented as a function of perturbation frequency in Figure 4. The perturbation amplitude is $a=4.0 \times$ $10^{-6} \mathrm{rad}$ for all simulations and is selected in the area where torque/angular velocity is linear and the value is selected in order to separate the response from numerical noise. One can see that it is difficult to identify the coefficients as stated in (17). There are two possible explanations to this: the coefficients depend on frequency and the stiffness is probably small due to the incompressible fluid. The stiffness is therefore assumed to be negligible $\left(K_{\text {Fluid }}=\right.$ 0 in (17)) in the analysis below. The added polar inertia is presented in Figure 5 and the added damping in Figure 6.

The later coefficients are added to the mechanical system, that is, (2). The polar inertia of the mechanical system is $J_{P}=1.57 \mathrm{Nms}^{2}$, the damping is $C=0 \mathrm{Nms}$, and the stiffness is $K=49000 \mathrm{Nm}$. In Figure 7 the reduced eigenfrequencies (18) and in Figure 8 the damping ratio (19) due to the flow for such a fluid-mechanical system are presented and the influence of the different coefficients is illustrated.

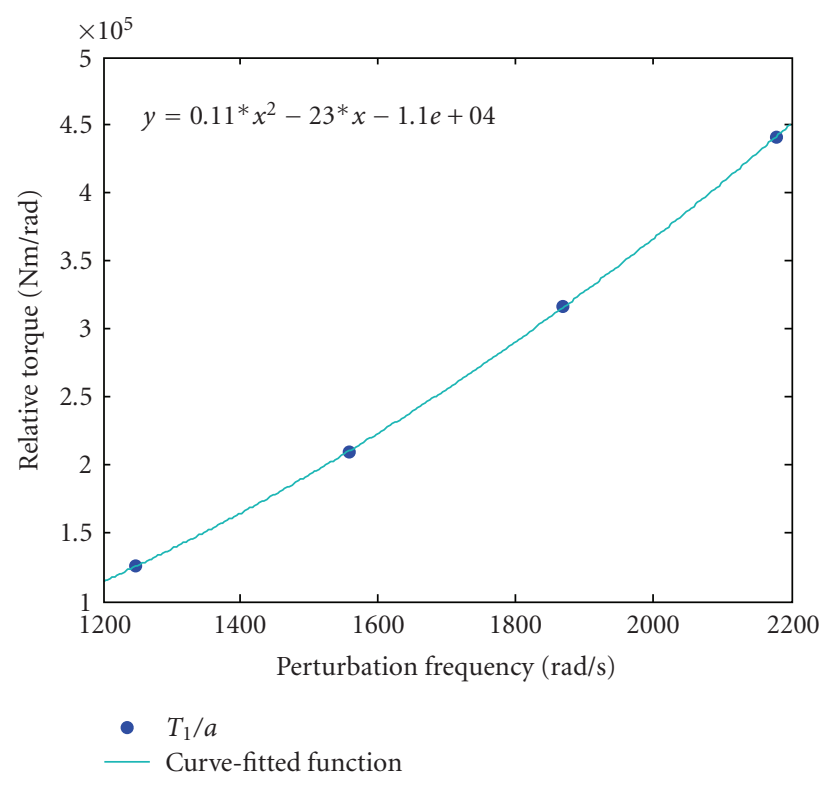

FIGURE 4: Identification of the coefficients of (17), together with a curve-fitted function (rotational speed is $52 \mathrm{rad} / \mathrm{s}$ ).

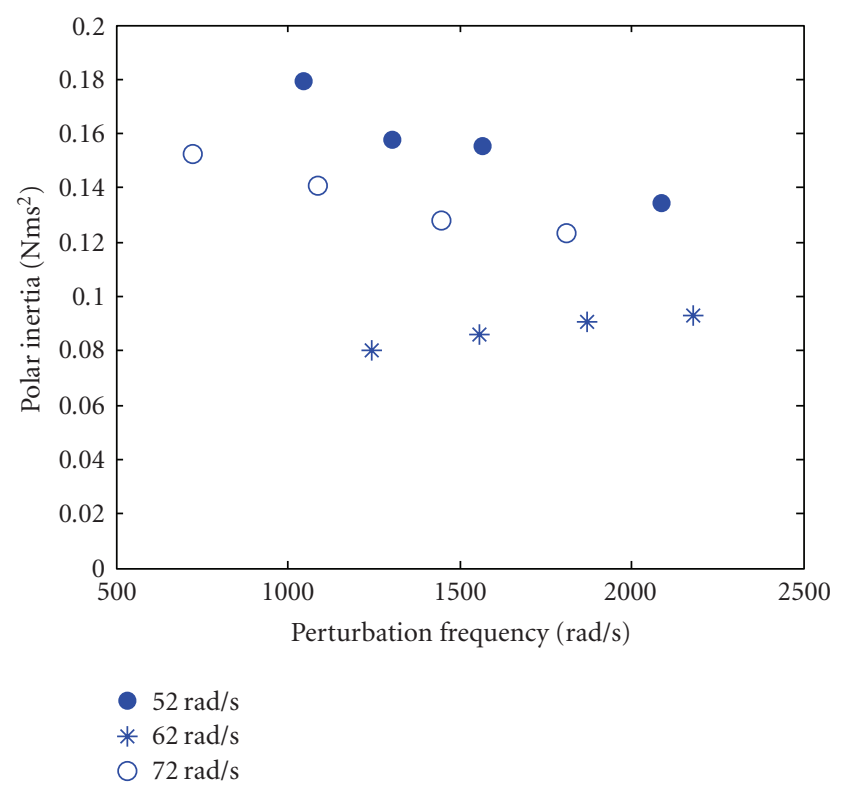

FIGURE 5: Additional polar inertia as a function of perturbing frequency and operating condition.

\section{Discussion}

Both added polar inertia and damping have a significant effect on the eigenfrequency of the mechanical system. The added polar inertia decreases the eigenfrequency $3-5 \%$ for all cases (see Figure 7). Concerning the damping, an additionally decrease of the eigenfrequency of $5-60 \%$ is observed (see Figure 7). One can see that both damping and polar inertia increases for off-nominal speed and with frequency. Recent research by Liang et al. [18], and Rodriguez et al. [19] has 


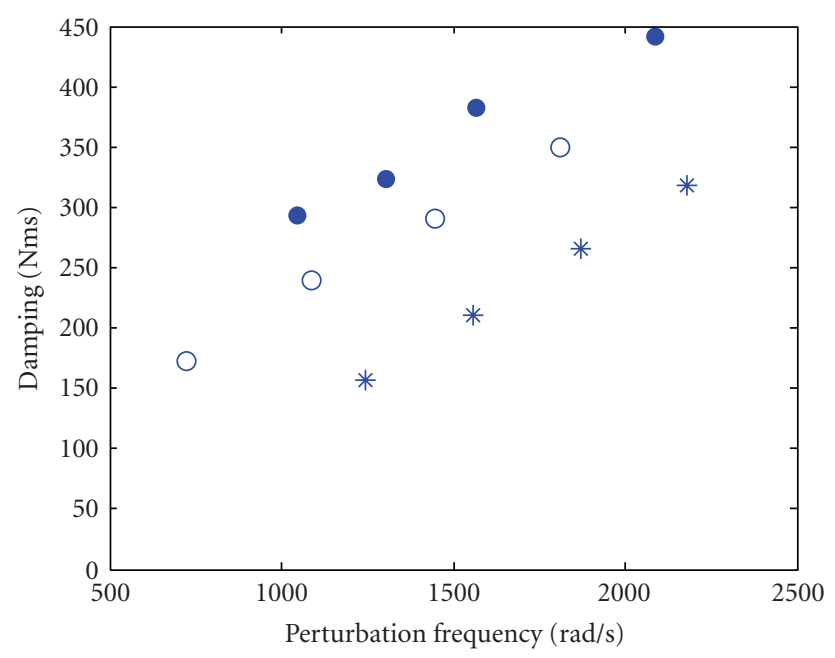

$52 \mathrm{rad} / \mathrm{s}$
* $62 \mathrm{rad} / \mathrm{s}$
$72 \mathrm{rad} / \mathrm{s}$

Figure 6: Additional damping as a function of perturbing frequency and operating condition.

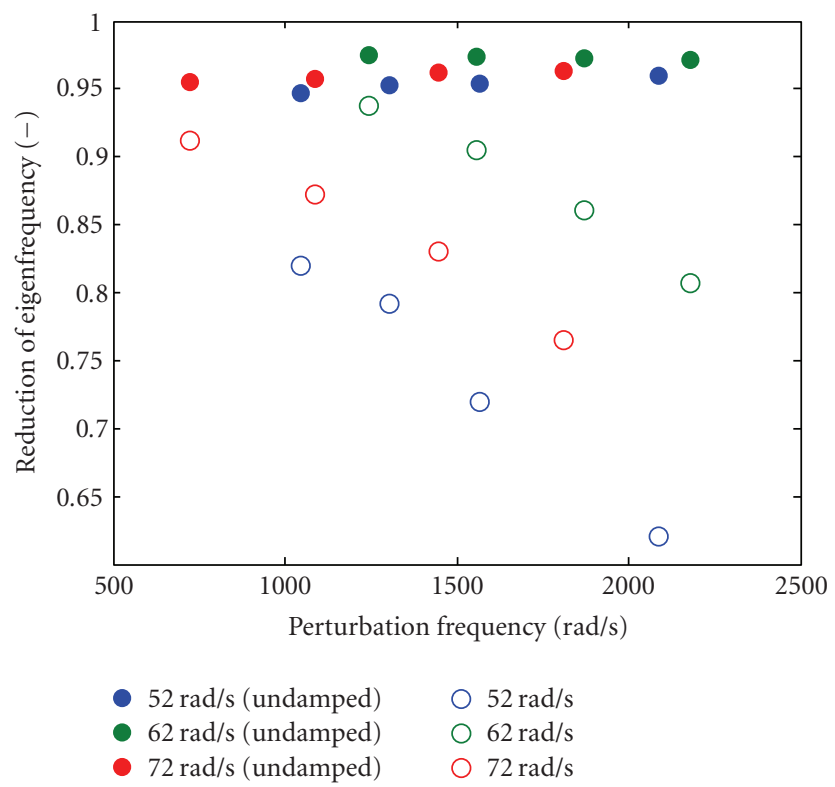

FIGURE 7: Reduction of the eigenfrequency (the eigenfrequency of the mechanical system is 1 ) due to the flow through the turbine. The "undamped" markers represent the effect of an added polar inertia alone.

shown that the eigenfrequencies are reduced by $10-39 \%$ for a nonrotating Francis runner in still water. The effect of added inertia in these papers are significantly higher than the case of nominal operating condition in the present work and the authors observe no strong effect of damping. An explanation to the difference between the present study and the earlier work is the dependency of frequency for both added inertia

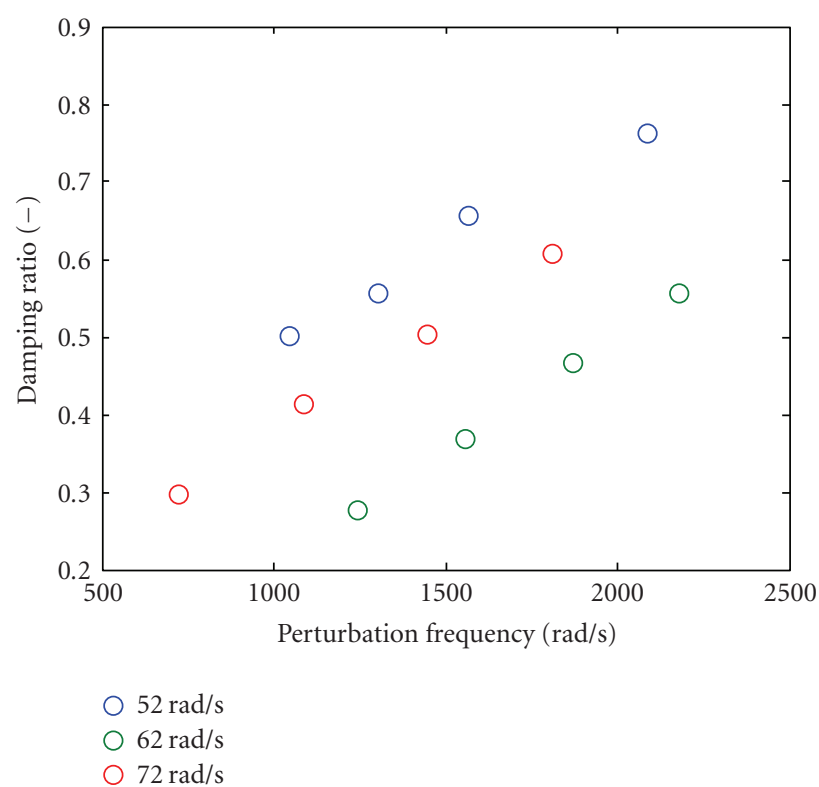

FIGURE 8: Additional damping due to the flow through the turbine (the damping of the mechanical system is zero).

and damping and that the present work includes the turbine flow.

Iso-surfaces are here used to illustrate the difference between the different operating conditions. Figure 9 shows iso-surfaces of regions where the turbulent kinetic energy is high. In Figures 10, 11, and 12 smearlines at the blades are presented in order to see the details of the flow.

The difference in the rotating speed results in different flow conditions for the different operating conditions. The guide vane angle is equal for all cases. Hence, the angle of attack at the leading edge of the runner blades is changed when changing the rotational speed. The tipclearance flow from the pressure side to the suction side is increased when the rotational speed is reduced. For high rotational speeds there is also a tip vortex at the runner blade pressure side due to the unfavorable angle of attack close to the tip. The tip vortex flow is the reason to the high turbulent kinetic energy near the tipclearence, which is shown in Figure 9. Figure 9 also shows high turbulence kinetic energy in the flow stagnation at the leading edges of the runner blades, and in separation regions. A major difference in the level of turbulence kinetic energy can be found below the runner cone in the recirculation region. The significant differences of the flow field for the different cases are also illustrated by the smearlines in Figures 10, 11, and 12. Figures 10 and 12 show a large non-axisymmetric recirculation area below the cone. The wakes below the runner vanes are also shown on the cone as well as the tipvortex flow. Figure 11 shows a small axisymmetric recirculation area below the cone.

Recent research of added mass of a cylinder by Wang et al. [24] has shown that the added mass is dependent on the velocity around a cylinder. The same effect is suspected in the present study, where the flow velocity differs between the cases. 


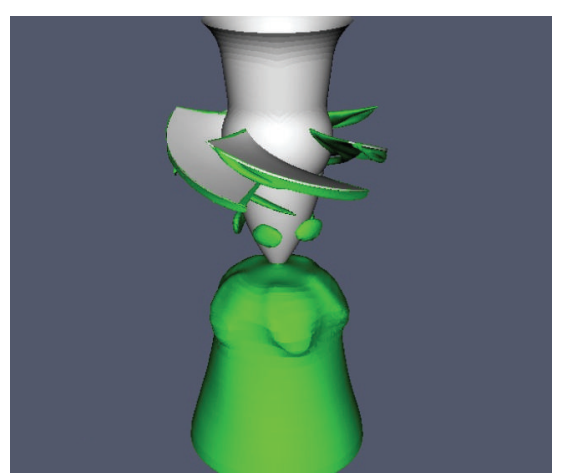

(a)

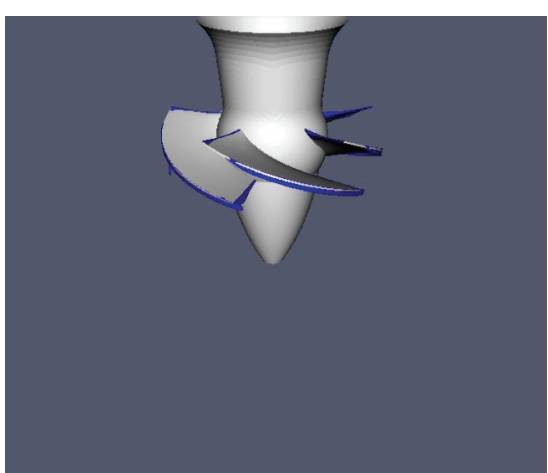

(b)

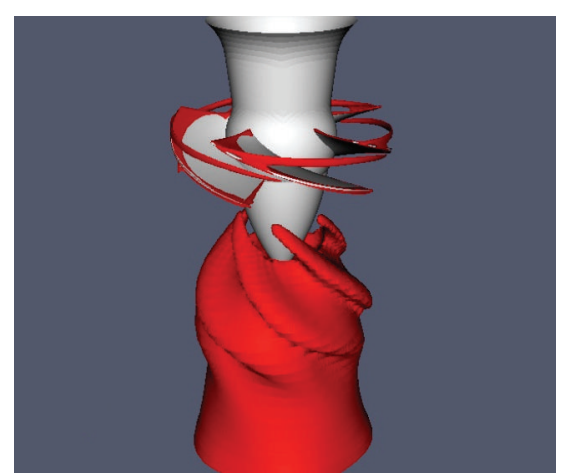

(c)

Figure 9: Iso-surface of turbulent kinetic energy, (a) $52 \mathrm{rad} / \mathrm{s}$, (b) $62 \mathrm{rad} / \mathrm{s}$, and (c) $72 \mathrm{rad} / \mathrm{s}$.

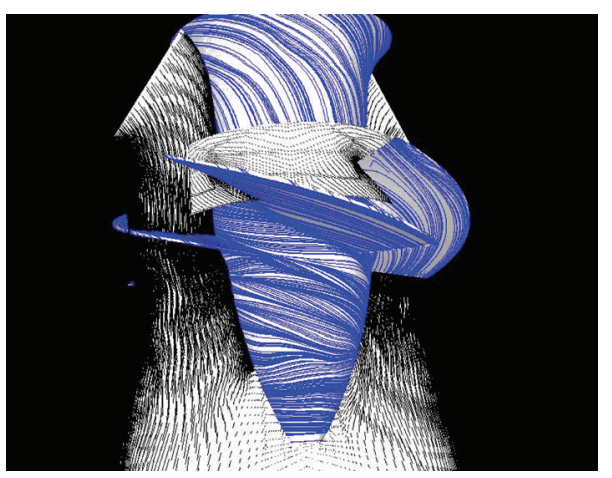

FIGURE 10: Smearlines and velocity vectors for $52 \mathrm{rad} / \mathrm{s}$.

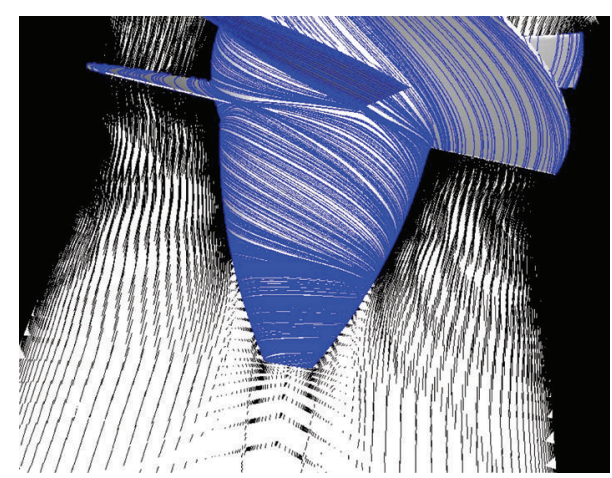

FIGURE 11: Smearlines and velocity vectors for $62 \mathrm{rad} / \mathrm{s}$.

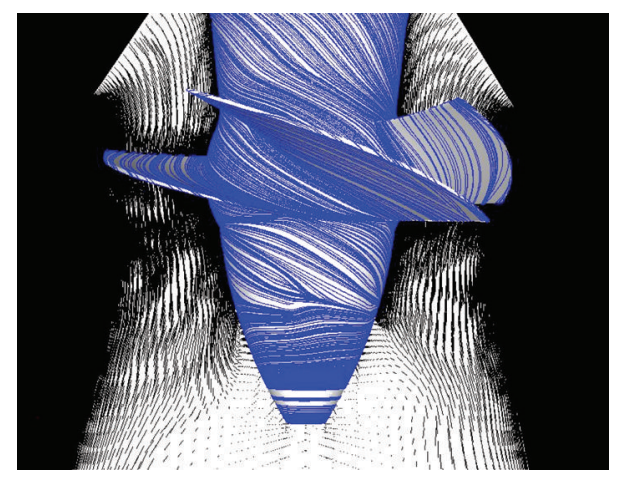

FIGURE 12: Smearlines and velocity vectors for $72 \mathrm{rad} / \mathrm{s}$.

\section{Conclusions}

The added polar inertia and damping due to the hydraulic system significantly affect the mechanical system. This results in a reduced eigenfrequency of $5-65 \%$ and an increase in the damping of $30-80 \%$. It is further concluded that the added coefficients are dependent on the turbine load and oscillating frequency. A change in the system properties of the mechanical system is important to consider in design and operation. Future studies should include experimental verification of the results in the present work.

\section{Nomenclature}

$\theta: \quad$ Angular displacement (rad)

$\omega_{D}: \quad$ Damped natural frequency $(\mathrm{rad} / \mathrm{s})$

$\zeta: \quad$ Damping ratio $(-)$

9: $\quad$ Prescribed frequency $(\mathrm{rad} / \mathrm{s})$

$\Omega: \quad$ Rotational speed ( $\mathrm{rad} / \mathrm{s})$

$\vec{n}_{\text {face }, i}:$ Normal vector at one face (-)

$p_{\text {face }, i}:$ Pressure one face $\left(\mathrm{N} / \mathrm{m}^{2}\right)$

$t: \quad$ Time (s)

$A_{\text {face }, i}:$ Area of one face $(\mathrm{Nm})$

$C: \quad$ Damping $(\mathrm{Nms} / \mathrm{rad})$

$C_{\text {Fluid: }}$ Added damping $(\mathrm{Nms} / \mathrm{rad})$

$\vec{F}_{\text {face }, i}:$ Force on one face $(\mathrm{N})$

$J_{P}: \quad$ Polar moment of inertia $\left(\mathrm{kgm}^{2}\right)$

$J_{P, \text { Fluid }}:$ Added Polar moment of inertia $\left(\mathrm{kgm}^{2}\right)$

$K: \quad$ Stiffness $(\mathrm{Nm} / \mathrm{rad})$

$K_{\text {Fluid }}$ : Added stiffness $(\mathrm{Nm} / \mathrm{rad})$

$M(t)$ : External moment $(\mathrm{Nm})$

$\vec{M}_{\text {face }, i}$ : Moment at one face $(\mathrm{Nm})$

$T^{\prime}(t)$ : Total torsional torque due to flow $(\mathrm{Nm})$

$T_{1,2}$ : Sine and cosine components of the torque $(\mathrm{Nm})$

$T_{\text {Amp }}$ : Amplitude of the oscillating part of the torque (Nm)

$T_{\text {Mean }}$ : Constant part of the torque $(\mathrm{Nm})$ 


\section{Acknowledgments}

The research presented in this paper has been carried out with funding by Elforsk $\mathrm{AB}$ and the Swedish Energy Agency through their joint Elektra programme and as a part of the Swedish Hydropower Centre (SVC) (http://www.svc.nu/). SVC has been established by the Swedish Energy Agency, Elforsk, and Svenska Kraftnät together with Luleå University of Technology, The Royal Institute of Technology, Chalmers University of Technology, and Uppsala University. Computations have been carried out with support from the Swedish National Infrastructure for Computing on the Hive and Ada clusters at C3SE, Chalmers University of Technology.

\section{References}

[1] J. J. Thomas, "Instabile Eigenschwingungen von Turbinenlaufen, Angefacht durch die Spaltstromnungen in Stopfbuchen und Beschau ungen," AEG-Sonderdruck, pp. 10391063, 1958.

[2] J. S. Alford, "Protection turbomachinery from self-excited rotor whirl," Journal of Engineering for Power, pp. 333-335, 1965.

[3] K. Urlichs, "Leakage flow in thermal turbo-machines as the origin of vibration-exciting lateral forces," Tech. Rep. NASA TT-17409, NASA, Washington, DC, USA, March 1977.

[4] H. W. Iversen, R. E. Rolling, and J. J. Carlson, "Volute pressure distribution, radial forces on the impeller and volute mixing losses of a radial flow centrifugal pump," ASME Journal of Engingeering for Power, vol. 82, pp. 136-144, 1960.

[5] A. Agostinelli, D. Nobles, and C. R. Mockridge, "An experimental investigation of radial thrust in centrifugals pumps," ASME Journal of Engineering for Power, vol. 82, pp. 120-126, 1960.

[6] G. T. Csanady, "Radial forces in a pump caused by volute casing," ASME Journal of Engineering for Power, vol. 84, pp. 337-340, 1962.

[7] P. Hergt and P. Krieger, "Radial forces in centrifugal pumps with guide vanes," Proceedings of the Institution of Mechanical Engineers, vol. 184, part 3N, pp. 101-107, 1969-1970.

[8] J. Colding-Jorgensen, "The effect of fluid forces on rotor stability of centrifugal compressors and pumps," in Proceedings of the 1st Workshop on Rotordynamic Instability Problems in High-Performance Turbomachinery, pp. 249-266, Texas A\&M University, College Station, Tex, USA, May 1980, NASA Conference Publication no. 2443.

[9] D. R. Adkins, Analysis of hydrodynamic forces on centrifugal pump impellers, Ph.D. thesis, California Institute of Technology, Pasadena, Calif, USA, 1985.

[10] D. R. Adkins and C. E. Brennen, "Analysis of hydrodynamic radial forces on centrifugal pump impellers," ASME Journal of Fluid Engingeering, vol. 110, pp. 20-28, 1988.

[11] U. Bolleter, A. Wyss, I. Welte, and R. Stürchler, "Measurement of hydrodynamic interaction matrices of boiler feed pump impellers," Journal of Vibration, Acoustics, Stress, and Reliability in Design, vol. 109, no. 2, pp. 144-151, 1987.

[12] U. Bolleter, E. Leibundgut, R. Stürchler, and T. McCloskey, "Hydraulic interaction and excitation forces of high head pump impellers," in Proceedings of the 3rd Joint ASCE/ASME Mechanics Conference, vol. 81, pp. 187-193, UCSD, San Diego, Calif, USA, July 1989.
[13] D. W. Childs, "Fluid-structure interaction forces at pumpimpeller-shroud surfaces for rotordynamic calculations," Journal of Vibration, Acoustics, Stress, and Reliability in Design, vol. 111, no. 3, pp. 216-225, 1989.

[14] F. J. Dietzen and R. Nordmann, "Calculating rotordynamic coefficients of seals by finite-difference techniques," Journal of Tribology, vol. 109, no. 3, pp. 388-394, 1987.

[15] T. Suzuki, K. Yonezawa, H. Horiguchi, T. Tsukiya, Y. Taenaka, and Y. Tsjuimoto, "A numerical analysis of rotordynamic uid forces on an artificial heart pump impeller in whirling motion," in Proceedings of the 12th International Symposium on Transport Phenomena and Dynamics of Rotating Machinery, Honolulu, Hawaii, USA, February 2008, paper-ID ISROMAC12-2008-20232.

[16] A. Ruprecht, C. Bauer, and M. Heitele, "Unsteady forces on the blading of an axial turbine caused by stator-rotor interaction," in Proceedings of the 9th IAHR WG International Meeting on the Behavior of Hydraulic Machinery under Steady Oscillatory Conditions, Brno, Czech Republic, September 1999.

[17] A. Ruprecht, M. Heitele, T. Helmrich, W. Moser, and T. Aschenbrenner, "Numerical simulation of a complete francis turbine including unsteady rotor/stator interactions," in Proceedings of the 20th IAHR Symposium on Hydraulic Machinery and Systems, pp. 1-8, Charlotte, NC, USA, August 2000.

[18] Q. W. Liang, C. G. Rodriguez, E. Egusquiza, X. Escaler, M. Farhat, and F. Avellan, "Numerical simulation of uid added mass effect on a francis turbine runner," Computers \& Fluids, vol. 36, pp. 1106-1118, 2007.

[19] C. G. Rodriguez, E. Egusquiza, X. Escaler, Q. W. Liang, and F. Avellan, "Experimental investigation of added mass effects on a Francis turbine runner in still water," Journal of Fluids and Structures, vol. 22, no. 5, pp. 699-712, 2006.

[20] M. Karlsson, H. Nilsson, and J.-O. Aidanpää, "Influence of inlet boundary conditions in the predictions of rotor dynamic forces and moments for a hydraulic turbine using CFD," in Proceedings of the 12th International Symposium on Transport Phenomena and Dynamics of Rotating Machinery, Honolulu, Hawaii, USA, February 2008, paper-ID ISROMAC12-200820078.

[21] H. Nilsson, "Evaluation of OpenFOAM for CFD of turbulent flow in water turbines," in Proceedings of the 23rd IAHR Symposium on Hydraulic Machinery and Systems, Yokohama, Japan, October 2006.

[22] H. Nilsson and L. Davidson, "A numerical investigation of the flow in the Wicket gate and runner of the Hölleforsen (Turbine-99) Kaplan turbine model," in Proceedings of the Turbine-99-Workshop 2: The 2nd ERCOFTAC Workshop on Draft Tube Flow, Älvkarleby, Sweden, June 2001.

[23] H. Jasak, H. G. Weller, and A. D. Gosman, "High resolution nvd differencing scheme for arbitrarily unstructured meshes," International Journal for Numerical Methods in Fluids, vol. 31, no. 2, pp. 431-449, 1999.

[24] Y. Wang, W. Chen, and M. Lin, "Variation of added mass and its application to the calculation amplitude response for a circular cylinder," China Ocean Engineering, vol. 21, no. 3, pp. 429-437, 2007. 

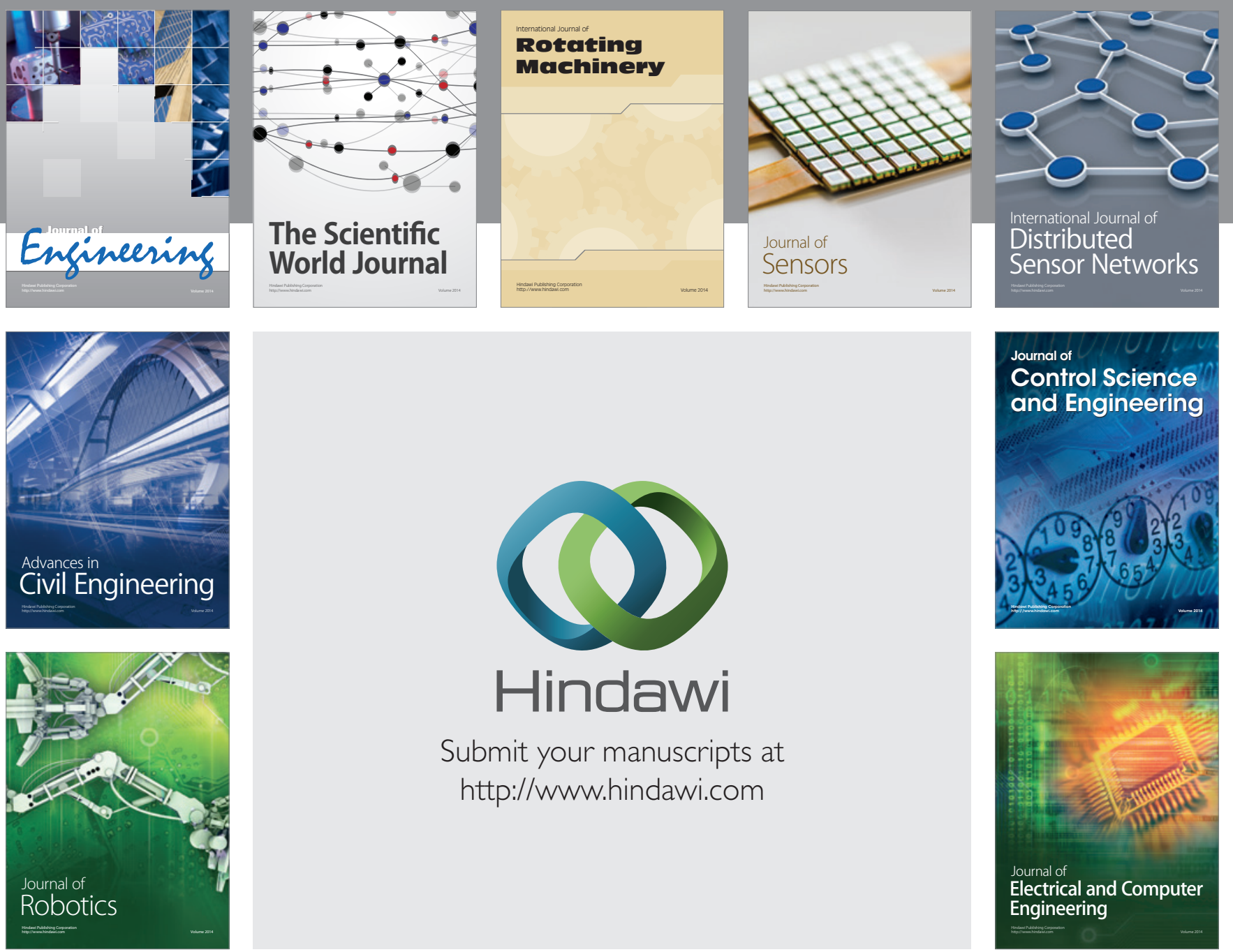

Submit your manuscripts at

http://www.hindawi.com
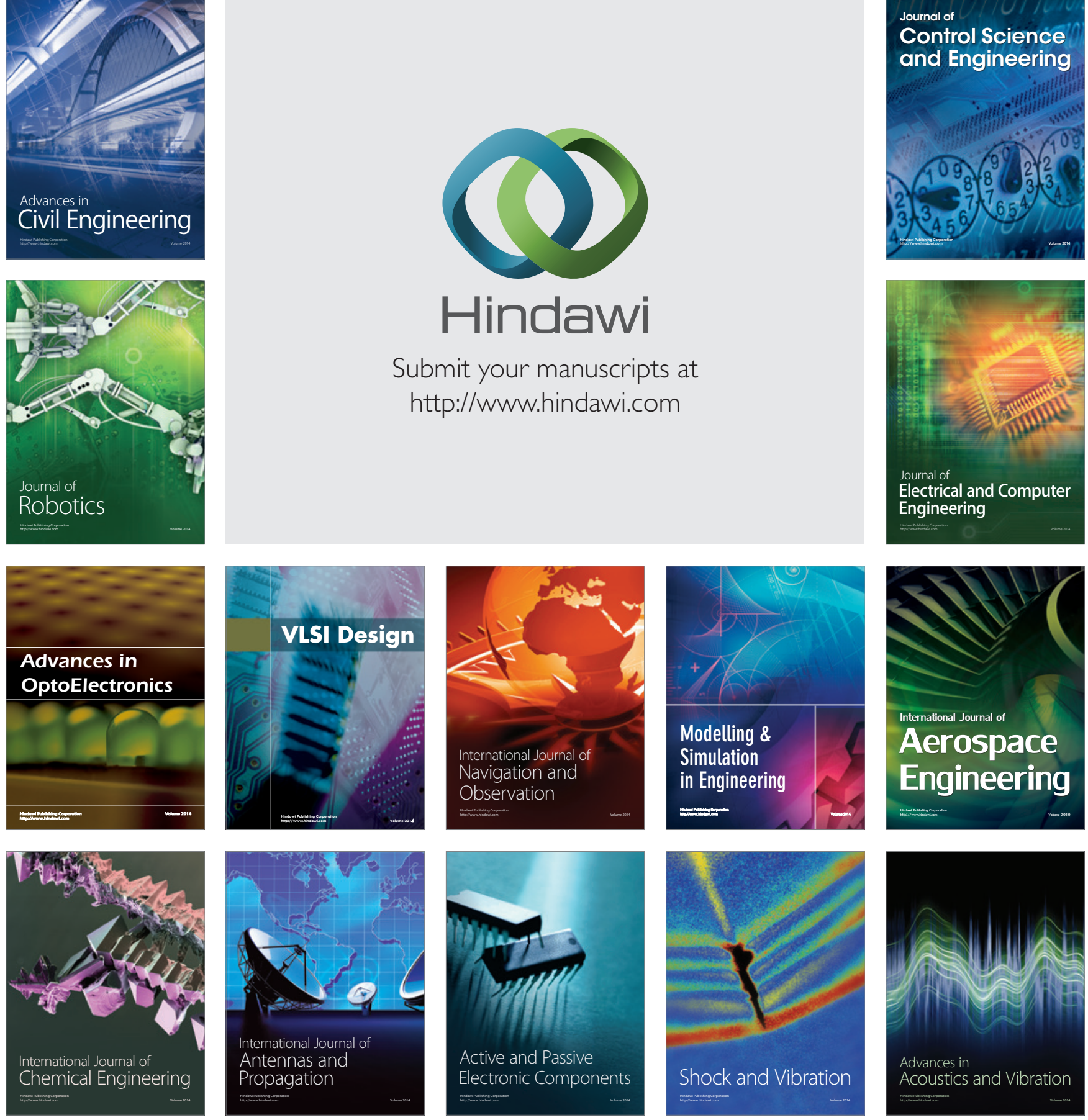